\title{
Nietzsche y el posthumanismo ${ }^{1}$
}

\section{Nietzsche and Posthumanism}

\author{
Vanessa Lemm ${ }^{2}$ \\ Universidad de Deakin (Australia) \\ ORCID: https://orcid.org/0000-0002-8444-0470
}

Recibido: 11-02-2021

Aceptado: 19-02-2021

\section{Resumen}

En Más allá del bien y del mal Nietzsche concibe la naturalización del ser humano, su retraducción en la naturaleza, como una experiencia liberadora en la que éste redescubre la naturaleza como una fuerza creativa y transformadora incorporada en él. Para Nietzsche, la cuestión del futuro del ser humano depende de su capacidad de re-incorporar la naturaleza. La naturaleza humana no es para Nietzsche algo dado del cuerpo (humano), algo que pertenece al ser humano per se. En cambio, la naturaleza en el ser humano viene con la tarea de retraducir, reinstalar, re-incorporar la naturaleza. Por ello, el pensamiento de Nietzsche sobre la

\footnotetext{
${ }^{1}$ Este artículo está basado en "Introduction: Who is Nietzsche's Homo Natura" y "Conclusion: Posthumanism and Community of Life" en V. Lemm, Homo Natura: Nietzsche, Philosophical Anthropology and Biopolitics, (Edinburgh, 2020), pp. 1-12 y 167-182.

${ }^{2}$ (vanessa.lemm@deakin.edu.au) Profesora de filosofía y Decana de la Facultad de Artes y Educación en la Universidad de Deakin, Australia. Anteriormente fue la primera Vicepresidenta y Decana del Colegio de Humanidades, Artes y Ciencias Sociales de la Universidad de Flinders (20182021), así como Directora de la Escuela de Humanidades y Lenguajes en la Universidad de New South Wales (2012-2018). En Chile, también ejerció como Directora del Instituto de Humanidades, de la Universidad Diego Portales (2009-2012).

Con una formación de perfil internacional (Doctorado en Filosofía en la New School for Social Research en Nueva York, Maestría en el King's College University de Londres y DEA en la Universidad de París, París I Panthéon-Sorbonne), ha ejercido cargos académicos en Estados Unidos, Alemania y Chile. Fue profesora invitada con financiación del Deutsche Akademische Austauschdienst (DAAD) y es miembro de la Royal Society de New South Wales. Su investigación aborda la relevancia de la filosofía de Nietzsche para los debates contemporáneos en biopolítica, posthumanismo y estudios animales. Ha publicado recientemente Homo Natura: Nietzsche, Philosophical Anthropology and Biopolitics (EUP, 2020). También es autora de Nietzsche y el pensamiento contemporáneo (Fondo de Cultura Económica, 2011) y de Nietzsche's Animal Philosophy: Culture, Politics and the Animality of the Human Being (Fordham, 2009). Ha publicado 5 volúmenes editados y numerosos artículos académicos y capítulos de libros sobre Nietzsche y el pensamiento político contemporáneo. Desde 2017, es editora de Nietzsche-Studien (De Gruyter).
} 
naturaleza está orientado hacia el futuro, abriendo el horizonte de la transformación humana. En este artículo sostengo que el pensamiento de Nietzsche sobre el homo natura es claramente posthumano y como tal sienta las bases para los debates contemporáneos sobre el posthumanismo.

Palabras-clave: Homo natura, posthumanismo, naturalización, Nietzsche.

\begin{abstract}
In Beyond Good and Evil, Nietzsche envisages the naturalisation of the human being, its retranslation back into nature, as a liberating experience where the human being rediscovers nature as a creative and transformative force that the human being embodies. For Nietzsche, the question of the future of the human being is contingent on whether the human being is capable of re-embodying nature. Human nature for Nietzsche is not a given of the (human) body, something that belongs to the human being per se. Instead, nature in the human being comes with the task of retranslation, replanting, re-embodying nature. This is why Nietzsche's thinking about nature is future oriented, opening up the horizon of human transformation. In this paper, I argue that Nietzsche's thinking about homo natura is distinctly posthuman and as such sets the stage for contemporary debates on posthumanism.
\end{abstract}

Key-words: Homo natura, Posthumanism, Naturalisation, Nietzsche.

Kant inauguró la época de las ciencias humanas al colocar por encima de sus tres famosas preguntas, “QQué puedo saber?”, “QQué debo hacer?”, “QQué me cabe esperar?”, la pregunta general: “¿Qué es el hombre?” (Kant 2000: 92). Foucault concluyó su crítica de las ciencias humanas anunciando "la inminencia de la muerte del hombre" (Foucault 2007: 332). Entremedio, y preparando el camino de uno al otro, se sitúa el famoso aforismo 230 de Más allá del bien y del mal. Preludio para una filosofía del futuro, en el que Nietzsche ofrece una respuesta a la pregunta de Kant introduciendo el enigmático término homo natura $(M B M, 230)^{3}$. Para Kant, el ser humano era el legislador de la naturaleza. Por el contrario, a lo largo de sus escritos Nietzsche insiste en la continuidad entre la naturaleza y el ser humano, y en la imposibilidad de alcanzar una posición trascendental por fuera de la naturaleza. Aun así, esto no significa que

\footnotetext{
${ }^{3}$ En este artículo utilizo las siguientes abreviaciones de los escritos de Nietzsche: $M B M=$ Más allá del bien y del mal; $H V=$ Sobre la utilidad y el perjuicio de la historia para la vida; EA = El Anticristo; $G C=$ La ciencia jovial (la $"$ gaya scienza $) ; V M=$ Sobre verdad y mentira en sentido extramoral .
} 
Nietzsche borre "el ser humano" en una especie de monismo ontológico. Más bien, sostengo que homo natura representa la fórmula paradojal en la que el humano es atrapado en un movimiento, en el cual mientras más natural es, más sobrehumano se vuelve. En este movimiento, la adopción de una "naturaleza más natural" $(H V, 10)^{4}$ del ser humano conduce a una superación del humano que está orientada hacia el devenir del sobrehumano.

Difícilmente hay un terreno interpretativo más disputado que el debate sobre el significado de homo natura y de términos relacionados como naturaleza, naturalidad, renaturalización (Vernatürlichung) y su importancia para el pensamiento de Nietzsche en su conjunto. Nietzsche presenta la realización del homo natura como "una tarea rara y loca (seltsame und tolle Aufgabe)" para "retraducir (zurückübersetzen) el hombre a la naturaleza" (MBM, 230). Hoy en día, las ciencias humanas inauguradas por Kant han retomado de manera entusiasta la tarea de Nietzsche, asumiendo la revolución darwinista y adoptando un giro evolucionista con la esperanza de producir hipótesis empíricamente verificables para explicar las manifestaciones de la vida humana. En sus formas evolucionistas y conductistas, el naturalismo causa furor en las ciencias humanas y, más recientemente, en filosofía. Mi reciente libro Homo Natura: Nietzsche, Philosophical Anthropology and Biopolitics ["Homo Natura: Nietzsche, antropología filosófica y biopolítica"] ofrece una nueva interpretación de la idea de homo natura en Nietzsche como una respuesta a la pregunta de Kant, que busca evitar el naturalismo reduccionista o cientificista. Para Nietzsche, la tarea de retraducir los seres humanos a la naturaleza debe ser realizada por científicos (Erkennende). Sin embargo, estos científicos tienen que distinguirse también por su probidad (Redlichkeit) y coraje para enfrentar "el terrible texto básico homo natura (schreckliche Grundtext homo natura)" $(M B M, 230)$. Mi libro intenta rastrear este terrible texto básico que cualifica la vida humana. La hipótesis central es que el texto básico de la naturaleza es aterrador en la medida que contiene lo que es incognoscible e indescubrible (desde una concepción positivista de la ciencia), algo de la naturaleza que está "más allá del bien y del mal" y que, cuando es admitido por la vida humana, transforma esta vida en algo creativo y así digno de afirmación.

En el aforismo 14 de El Anticristo, Nietzsche anuncia que ha "rectificado (umgelernt)" su manera de pensar la naturaleza humana y que la ha "reintegrado (zurückgestellt) en el mundo animal" $(E A, 14)$. Sostengo que el descubrimiento del homo natura no lleva a Nietzsche a desechar la historia y a adoptar una concepción científica de la evolución biológica que la reemplace. Más bien, la renaturalización del ser humano va de la mano con una renaturalización de la historia. Nietzsche se pregunta qué diferencia supone para nuestra autocomprensión histórica el descubrimiento del homo natura. ¿Qué significa el

\footnotetext{
${ }^{4}$ Traducción modificada.
} 
"retorno a la naturaleza" y la renaturalización del ser humano para nuestro entendimiento de la historia? Ofrezco dos posibles respuestas a esta pregunta: primero, nos permite escribir una historia natural basada en el cuerpo humano. Segundo, dicha historia natural revela una concepción de la naturaleza humana que está esencialmente involucrada en la (auto)transformación cultural, y como tal supera la dicotomía entre cultura y naturaleza. En mi trabajo, sostengo que para Nietzsche el ser humano pertenece enteramente a la naturaleza y es una parte inseparable de ella (Lemm 2010). Para responder la pregunta "¿Qué es el ser humano?", Nietzsche rechaza toda narrativa teleológica y no apela a la trascendencia de valores que caracteriza los enfoques metafísicos y religiosos del sentido de la vida humana. Para él, el ser humano no tiene esencia porque ser humano consiste en estar envuelto en un movimiento dialéctico tal que cuanto "más natural" intenta ser el ser humano, más capaz se vuelve de "superarse" a sí mismo.

Mi lectura de Homo Natura busca mostrar que esta condición sobrehumana es para Nietzsche una condición cultural de producción y creatividad artística que se alcanza a partir de una experiencia de la naturaleza como un caos de pulsiones y un abismo de conocimiento. Este logro está mediado por el cultivo de características que pertenecen a la vida animal y vegetal. Al reconocer la animalidad e incluso el aspecto vegetal del ser humano, Nietzsche pone estrechamente en relación la actitud de probidad científica que discierne un continuo entre naturaleza y cultura con una concepción ético-política de lo que es bueno por naturaleza, a la que los cínicos fueron los que más se acercaron. Para el "espíritu libre” o el científico probo de Nietzsche, la pregunta "¿Qué es el ser humano?” es lo mismo que la pregunta “¿Qué es natural para el ser humano?” o “QQuién es el ser humano natural?” Aunque Nietzsche se ocupa del humanismo anti-naturalista que él asocia con la cristiandad y extrae de su interpretación de los griegos la idea de que son los que más cerca llegan a desarrollar el ser humano natural, el punto de su enseñanza sobre el homo natura es mostrar que ningún pasado histórico de la especie humana puede formular la respuesta correcta a qué es natural en el ser humano. La razón es que, para él, lo que resulta natural en el humano es una capacidad de transformación y metamorfosis de su forma de ser. Según mi interpretación del homo natura, el llamado de Nietzsche a renaturalizar la especie humana conlleva en último término un descentramiento de su humanidad con relación al continuo de vida que es capaz de incorporar. En este sentido, la enseñanza de Nietzsche sobre el homo natura señala el advenimiento del posthumanismo. En este artículo busco explorar más a fondo la relación entre Nietzsche y los debates contemporáneos sobre el posthumanismo. Como discurso crítico, el posthumanismo contemporáneo reúne todos los motivos clave presentes ya en Nietzsche. Un rechazo del antropocentrismo y la jerarquía de especies basada en 
una idea de un continuo entre naturaleza y cultura; un rechazo del humanismo kantiano en favor de una visión transformadora y de auto-superación del humano; y por último, aunque no menos importante, un propósito normativo que busca redefinir la posibilidad de un sujeto activo en contraste con un mero atributo de adaptación a circunstancias dadas, sin el cual el posthumanismo dejaría de ser un discurso crítico (Braidotti 2016; Wolfe 2010). Sin embargo, a pesar que el discurso posthumanista tiene como precursor común el homo natura de Nietzsche, el posthumanismo también se halla dividido con relación a cómo recupera su legado nietzscheano anti-humanista y anti-antropocéntrico. En lo que sigue, ofrezco una posible representación de esta división, la que caracterizaré como una oposición entre posthumanismo biopolítico y posthumanismo de ensamblajes.

\section{Dos formas de posthumanismo}

Como ejemplo de posthumanismo de ensamblajes, consideraré el trabajo de Rosi Braidotti y de Cary Wolfe. A pesar de las diferencias entre las fuentes teóricas de sus respectivas versiones de posthumanismo -Gilles Deleuze para Braidotti, Jacques Derrida y Niklas Luhmann para Wolfe- ambos entienden el posthumanismo no tanto como la "borradura" del humano, sino como una nueva descripción de "la especificidad del humano -sus modos de ser en el mundo, sus modos de conocer, observar y describir- mediante (paradójicamente, para el humanismo) el reconocimiento de que es una creatura fundamentalmente prostética que ha co-evolucionado con varias formas de tecnicidad $\mathrm{y}$ materialidad, formas que son radicalmente 'no-humanas' y que sin embargo han hecho al humano ser lo que es" (Wolfe 2010: xxv) ${ }^{5}$. De manera similar, Braidotti sostiene que el posthumanismo es la simultánea adopción de dos proposiciones (Braidotti 2015). La primera puede encontrarse igualmente en Nietzsche: afirma que no existe una "humanicidad originaria" (Kirby 2011). La segunda, en cambio, no pertenece a Nietzsche: afirma que solo existe una "tecnicidad originaria" (MacKenzie 2006).

La segunda afirmación es fundamental para recientes elaboraciones del transhumanismo basado en la tecnología, tal como se encuentra en los discursos de Ray Kurzweil sobre la singularidad y Yuval Harari sobre Homo deus ${ }^{6}$. El posthumanismo inspirado en la cibernética y la Inteligencia Artificial sostiene que el progreso tecnológico transformará al ser humano en un superhombre o un sobrehumano. En cambio, yo he propuesto que la enseñanza de Nietzsche

\footnotetext{
${ }^{5}$ La cursiva es mía.

${ }^{6}$ Sobre la cuestión de si Nietzsche es un precursor o un enemigo del transhumanismo, ver Yunus Tuncel, ed., Nietzsche and Transhumanism: Precursor or Enemy (Cambridge, 2017).
} 
sobre el homo natura busca evitar todos y cada uno de los intentos por naturalizar el ser humano a través de un reduccionismo cientificista (Lemm 2016). Nietzsche evita este tipo de reduccionismos al ofrecer una visión de la verdad científica como probidad, la que implica afirmar la incognoscibilidad de la naturaleza humana, en la medida en que esta visión se basa en el carácter caótico y abisal de la propia naturaleza, y por ende en los límites de la ciencia humana. Para Nietzsche, la condición sobrehumana no se alcanza mediante la adición o el suplemento de tecnología al ser humano, sino que mediante el reconocimiento de la continuidad de la experiencia humana con la vida animal y vegetal. Es solo basándose en esta vida, en aquello que Ludwig Binswanger llama la "historia interna" de la incorporación vivida, que el ser humano se vuelve creativo (Lemm 2019).

La cuestión de la producción cultural humana lleva tanto a Nietzsche como a Freud de vuelta a los griegos y su concepción mitológica de la naturaleza como caos. Este retorno se refleja en la figura del sobrehumano en Nietzsche como un ejemplo de cómo uno podría concebir la (auto) transformación humana basada en la naturaleza como fuente de renovación cultural. Nietzsche concibe la naturalización del ser humano, su retraducción de vuelta en la naturaleza, como una experiencia liberadora en la que el ser humano redescubre la naturaleza como una fuerza creativa y transformadora que el ser humano puede incorporar. Nietzsche y Freud se preguntan bajo qué condiciones el ser humano puede volverse nuevamente humano. Para Nietzsche, la pregunta por el futuro del ser humano depende de si el ser humano es capaz de re-incorporar la naturaleza.

La posición del posthumanismo biopolítico, tal como es desarrollado a partir de la enseñanza de Nietzsche sobre el homo natura, entiende la tecnicidad o la tecnología como inherentemente inmunitaria. Desde esta perspectiva biopolítica, y empleando el concepto de Agamben, no hay anthropos sin una "máquina antropológica", que funciona separando una comunidad originaria de vida para la cual la distinción entre zoe y bios es insostenible, y recombinando zoe y bios en un aparato a través del cual el poder es ejercido sobre la vida (Agamben 2006). Desde esta perspectiva, el posthumanismo biopolítico responde con la recuperación de una comunidad de vida más allá de todo intento por inmunizar el ser de una especie contra otra. El posthumanismo biopolítico articula su anti-humanismo y su anti-antropocentrismo a través de una idea de vida común antes que en la afirmación del carácter originariamente prostético o de ensamblaje del ser humano.

La diferencia entre estas dos aproximaciones fundamentales al posthumanismo puede apreciarse observando el diferente rol y función que reciben la vida animal y vegetal en sus respectivas auto-comprensiones. Tanto para Braidotti como para Wolfe, en cierto sentido, la cuestión animal sólo se 
abre gracias a una comprensión cibernética de la vida como un sistema autoproductivo o autopoiético. En este sentido, no habría una "cuestión animal" sin plantear previamente la cuestión de la tecnología ${ }^{7}$. Como dice Braidotti, "la distancia que uno está dispuesto a tomar respecto del antropocentrismo también depende de la evaluación y la relación que uno establezca con los desarrollos tecnológicos contemporáneos. En mi trabajo, siempre he acentuado la dimensión tecnofílica y el potencial liberador e incluso transgresivo de estas tecnologías" (Braidotti 2016: 16) ${ }^{8}$. Para Braidotti -y lo mismo aplica para la adopción de la idea luhmanniana de la auto-referencia y la auto-clausura de los sistemas vivos por parte de Wolfe-, el continuo entre naturaleza y cultura, la posibilidad de una comunidad entre vida humana y no-humana, se basa en último término en lo que ella llama "la ubicuidad de la mediación tecnológica" (ibíd: 17). La afirmación fundamental de este posthumanismo de ensamblajes, según el cual la naturaleza humana es rechazada en favor del "sujeto posthumano como un ensamblaje compuesto de elementos humanos, no orgánicos, maquínicos y otros" (ibid.: 19), es el hecho trascendental de "la presencia estructural de prácticas y aparatos de mediación que inscriben a la tecnología como 'segunda naturaleza" (ibid. $)^{9}$. En otras palabras, y paradojalmente, el humano solo puede entrar en comunidad con una "primera naturaleza" separándose él mismo de ella a través de la tecnología como "segunda naturaleza".

Ni Braidotti ni Wolfe tematizan el hecho interesante de que el fundamento "técnico" o "prostético" de sus posthumanismos se deriva él mismo de una variante de antropología filosófica, específicamente, de la interpretación que hace Helmuth Plessner del homo natura de Nietzsche en términos de una vida animal que es radicalmente incompleta e insegura, pobre en instinto, y cuyo ser desde el principio está en necesidad de compensación: un ser que es "artificial por naturaleza" (Plessner 2003). Plessner extrae la idea del ser humano como un ser "deficiente" a partir de la crítica de Nietzsche del intelecto humano como un órgano insuficientemente desarrollado, cuyo único propósito es compensar la relativa debilidad del ser humano con relación a otros animales y a su entorno $(G S, 110$ y $V M)$. En Nietzsche, el intelecto ya

7 Wolfe atribuye a Luhmann, y a Maturana y Varela este enfoque cibernético de la vida, pero también se encuentra, por cierto, en el ideal del cyborg de Donna Haraway y luego, en las especies de compañía diseñadas tal como lo ejemplifica el OncoMouse y otras hibridaciones o formas de vida ensambladas de ese tipo (Haraway 2008). Una idea similar puede encontrarse también en el trabajo de Katherine Hayles (1999), otra precursora del discurso posthumanista contemporáneo.

${ }^{8}$ La cursiva es mía.

9 Ver también Braidotti (2015), donde ella afirma que "los confines entre las categorías de lo natural y lo cultural han sido desplazados y, en gran medida, esfumados por los efectos de los desarrollos científicos y tecnológicos" (Braidotti 2015: 13). Para otras declaraciones sobre el fundamento tecnológico del "continuo naturaleza-cultura", ver (ibid.: 77-78, 99-103, 123, 135, 163-164, 167, 188). En su artículo sobre la comunidad entre su posición y la de Haraway, Braidotti (2006) habla de un "rechazo a caer en la trampa de la división clásica naturaleza/cultura: no existe un telos u orden natural distinto de la mediación tecnológica" (Braidotti 2006: 199). 
no es el sello distintivo de la superioridad del ser humano sobre la naturaleza y de su ventaja técnica sobre la vida animal. Solo que en mi explicación del homo natura, la inseguridad del ser humano con relación a otras formas de vida ya no es el punto fundamental de la antropología filosófica de Nietzsche. Más bien, Nietzsche (así como Freud) presenta un discurso sobre la prioridad del cuerpo humano sobre el alma humana como órgano de conocimiento, mostrando al mismo tiempo que es la incognoscibilidad de los impulsos lo que otorga la capacidad transformativa del ser humano. La antropología filosófica de Nietzsche parte de lo que Binswanger llama "historia vital interior" antes que de la funcionalidad del cuerpo humano. Mientras que la tecnicidad busca siempre ya incrementar esta funcionalidad, ella tiene poco que decir sobre la auto-transformación cultural en la que se involucra el ser humano a través de su "historia interior" del cuerpo. Es más, para Nietzsche las raíces de la creatividad humana provienen de la "crueldad" de la animalidad tanto como del impulso de incorporación y reproducción característico de la vida vegetal: ninguna de estas características denota para Nietzsche una fuente de debilidad, de impotencia o de inseguridad.

El presupuesto del ser humano necesitado de un mecanismo de defensa suplementario es en sí mismo interior a la lógica inmunitaria de la tecnología. En mi opinión, Nietzsche (y Freud) busca superar la así llamada "debilidad" inducida por la lógica civilizacional que levanta barreras entre la vida humana (bios) y toda otra vida (zoe) apelando a una recuperación de la naturaleza como base de la creatividad y auto-superación humanas. Para Nietzsche, el problema de la civilización y la tecnología es que inmunizan al ser humano contra su propia naturaleza animal y vegetal, separando al ser humano de la comunidad de vida y por consiguiente de aquellos impulsos e instintos que de otro modo le permitirían involucrarse significativamente con otras formas de vida no humana.

\section{Zoe-igualitarismo y comunidad de vida}

La concepción del anti-humanismo de Deleuze y Guattari como una función del "devenir-animal" es claramente una de las construcciones conceptuales más dependientes de Nietzsche elaboradas por los filósofos franceses (Deleuze y Guattari 2002). Este elemento nietzscheano de Deleuze y Guattari es retomado en la proposición de Braidotti: "zoe, o la fuerza generativa de vida no humana" que "gobierna a través de una interconexión trans-especies y transgénica (...) que puede ser mejor descrita como una filosofía ecológica de sujetos no-unitarios incorporados y de pertenencias múltiples" (Braidotti 2013: 203). En Lo posthumano, Braidotti sostiene que el continuo entre naturaleza y 
cultura implica un "igualitarismo zoe-centrado" (Braidotti 2015: 77) ${ }^{10}$. Por el contrario, Wolfe rechaza tal igualitarismo ya que socavaría la idea misma de un sistema auto-referencial y auto-poiético. Tal vez no hay mejor manera de clarificar la diferencia entre el posthumanismo biopolítico y el de ensamblajes que analizando los argumentos a favor y en contra de la posibilidad de un zoeigualitarismo.

El zoe-igualitarismo que Wolfe se propone criticar en su Before the Law es aquel adoptado por Roberto Esposito y forma parte del enfoque afirmativo que propone de la biopolítica (Wolfe 2013). Al igual que Braidotti, Esposito también se basa en la idea de Deleuze de un devenir animal del ser humano, pero a diferencia de ella, lo entiende a través de un discurso sobre la comunidad y la inmunidad de la vida que se apoya en Nietzsche, y construye a partir de él lo que podría llamarse un posthumanismo biopolítico.

Esposito adopta dos principios: primero, el principio de la continuidad biológica de la vida, y segundo, la unidad y continuidad de la totalidad de la vida donde "ninguna porción de esta puede ser destruida en favor de otra: toda vida es forma de vida y toda forma de vida ha de referirse a la vida" (Esposito 2006: 312). Wolfe habla de un "principio de equivalencia ilimitada para cada forma de vida" (Wolfe 2013: 56). Ambos principios también se encuentran en el corazón de la concepción de Nietzsche del homo natura, como he sostenido a lo largo de mi libro. Para Esposito, al igual que para Nietzsche, no existe jerarquía entre formas de vida y todas las formas de vida deben ser afirmadas sin discriminación. Mientras que la biopolítica afirmativa de Esposito considera el zoe-igualitarismo como una contribución a la pluralización de formas de vida inherentemente singulares, Wolfe aboga por un enfoque "pragmático" que discrimine qué formas de vida pueden ser incluidas en la comunidad de vida humana y cuáles debieran ser excluidas.

Para Wolfe, existen ciertas formas de vida (viral, por ejemplo) que la forma humana de vida debe destruir activamente si pretende sobrevivir. Wolfe objeta la "adopción incondicional de toda forma de vida como sujetos de protección inmunitaria" y en cambio recomienda un retorno a una ética derridiana de la responsabilidad. Él es de la opinión que no todas las formas de vida "pueden ser bienvenidas, ni todas al mismo tiempo" (Wolfe 2013: 103), y que una ética derridiana de la responsabilidad podría proveernos de la herramienta correcta para distinguir entre aquellas formas de vida que caen bajo la protección de la ley y aquellas que permanecen "antes de la ley" (ibid.: 103-105). Aun así, sigue sin quedar claro cómo este enfoque pragmático podría prevenirnos de caer nuevamente en la distinción de la continuidad de la vida en términos de

${ }^{10}$ Van Ingen critica este igualitarismo sobre la base de que plegar la cultura a la naturaleza no permite la agencia humana, a menos que se crea que la naturaleza "por sí misma" pueda resolver problemas como el cambio climático, etc. (Van Ingen: 537).

Araucaria. Revista Iberoamericana de Filosofia, Política, Humanidades y Relaciones Internacionales, año $23, \mathrm{n}^{\circ} 46$. Primer cuatrimestre de 2021. Pp. 291-306. ISSN 1575-6823 e-ISSN 2340-2199 https://dx.doi.org/10.12795/araucaria.2021.i46.14 
una jerarquía de especies en la que algunas son más valoradas que otras, en la que algunas son más bienvenidas que otras, en la que algunas se extinguen por el bien de la supervivencia de otras.

A diferencia del posthumanismo de ensamblajes y de su hipótesis prostética, Esposito acude a Nietzsche y su concepción de la vida con el fin de deshacer lo humano como resultado de dispositivos inmunitarios, o en términos de Agamben, para romper la "máquina antropológica" (Agamben 2006). En Bios, Esposito (2006) identifica simultáneamente en Nietzsche la expresión de una tendencia autodestructiva presente en la maximización de la inmunidad y de lo que llama "hiperinmunidad", y una vía más afirmativa en la que la inmunidad vuelve a ser el custodio y productor de la vida. En esta perspectiva, la renaturalización del ser humano implica una apertura del individuo hacia aquello que constituye una amenaza para él con el fin de aliviar la sujeción que la propia auto-protección ejerce sobre el individuo. Los humanos necesitan inmunizarse a sí mismos mediante un retorno a la animalidad y necesitan romper todas las ilusiones artificiales y espirituales con las que han buscado separarse a sí mismos con fines de auto-preservación de otras especies vivas. Al romper las barreras de la civilización, los humanos se redescubrirían como parte de una comunidad de vida que admite la vida humana entre otras formas de vida no humana.

Una biopolítica afirmativa reconoce que la vida como devenir se forma y transforma, se crea y recrea a sí misma en y a través de sus múltiples encuentros con otras formas de vida. La vida es creativa, se da a sí misma una forma y crea y recrea activamente sus diversas formas y modos de vida. En mi libro Homo Natura, muestro que estas características esenciales de la vida se encuentran en el centro de la concepción transformadora del homo natura de Nietzsche. La naturaleza humana refleja la vida en la medida en que no sólo es excedente, plenitud y sobreabundancia sino también creatividad, normatividad y creación de valor. Nuevamente este es un punto importante ya que muestra por qué una biopolítica afirmativa refuta la visión según la cual existe una perspectiva externa y por encima de la vida que "da" valor, "permite" la vida o "asigna" el derecho a vivir, como sostiene Wolfe. Por el contrario, vida y valor, vida y norma, vida y forma son inseparables la una de la otra en la medida que valor, norma y forma son inherentes a la vida, son expresiones de la creatividad de la vida.

\section{Comunidad de vida y biopolítica afirmativa}

La biopolítica afirmativa tiene una importante contribución que hacer al debate sobre la inclusión y exclusión de la vida dentro de la ley. En primer 
lugar, la biopolítica afirmativa ofrece una idea de la política orientada hacia el devenir de la comunidad. En este caso, comunidad significa una experiencia de lo común, en la que esto último no designa una identidad compartida o una experiencia común basada en alguna especie de sentimiento o pertenencia comunitaria que elimina las diferencias, sino más bien designa la comunidad de aquello que es inherentemente plural y singular. Desde la perspectiva de la biopolítica afirmativa, la comunidad sólo es posible para aquello que es diferente y plural, singular e "impersonal" como plantea Esposito. Como tal, la biopolítica afirmativa no es una política del "sujeto de protección inmunitaria" como sostiene Wolfe (2013: p. 55), sino una política de la comunidad que es una política de "pura relación", una "relación sin sujeto" (Esposito 2006: 142-143).

En segundo lugar, esta lucha por la comunidad viene de la mano con una lucha por la justicia. La justicia no refiere a una relación recíproca, contractual, entre iguales basada en una economía del intercambio, que iría en el mejor interés de las dos partes. En lugar de ello, la justicia designa una relación asimétrica de donación que es inherentemente a-económica y en la que aquello que nos une mutuamente es el hecho que estamos en deuda los unos con los otros. Estamos vinculados unos a otros por una infinita responsabilidad hacia el otro, una deuda infinita que nunca podrá agotarse. Somos iguales a la luz de esta deuda que pesa sobre nosotros. Desde la perspectiva de una biopolítica afirmativa, la justicia se basa en la donación y el gasto. Ella es a-liberal y antiutilitaria.

Hasta aquí y en términos muy generales, esta concepción de la comunidad y de la justicia se articula a través del nuevo pensamiento de la comunidad en la filosofía continental, desde Georges Bataille y Maurice Blanchot hasta JeanLuc Nancy y Giorgio Agamben. Lo que distingue a estas formas de comprender la comunidad, es que el vínculo común lo provee principalmente el lenguaje, independientemente de que por lenguaje se entienda un idioma práctico dado, hablado y vivido, o que se entienda por ello una entidad ontológica, el lenguaje como la "morada del ser" según Heidegger (2006) ${ }^{11}$. Al contrario, en la

${ }^{11}$ El nuevo pensamiento de la comunidad es en muchos sentidos una respuesta a la crisis del humanismo en el siglo XX. Sin embargo, hasta el día de hoy este pensamiento no problematiza de manera suficiente la precaria frontera entre formas de vida humana y no humana. Se pueden distinguir dos momentos en el desarrollo del debate sobre la crisis del humanismo en el siglo XX. El primer momento es ejemplificado por el intercambio entre Sartre y Heidegger en los años 1940. Concierne las diferentes recepciones de la temprana analítica de la existencia humana (Dasein). En este primer momento, la existencia (Dasein) del ser humano, en sus múltiples sentidos, es considerada la responsable del descentramiento del sujeto humano. El primer momento del debate sobre la crisis del humanismo fue seguido por las críticas estructuralistas y post-estructuralistas de los años 1960 realizadas por Lévi-Strauss, Derrida y Foucault. Puede argumentarse que este segundo momento del debate es el resultado de la recepción del Heidegger tardío y de su "giro (Kehre)" respecto de la prioridad de la existencia humana hacia la prioridad del lenguaje como emplazamiento de la posibilidad de la ontología. De acuerdo con este segundo debate sobre la crisis del humanismo, lo 
biopolítica afirmativa, el vínculo común lo provee una concepción nietzscheana de la vida entendida como una vida compartida que funciona como la fuerza unificadora entre diferentes formas de vida. La biopolítica afirmativa ofrece así una manera de pensar juntas comunidad y vida. La enseñanza de Nietzsche sobre el homo natura aporta una nueva perspectiva sobre el pensamiento actual de la comunidad al señalar que la sociedad humana siempre procede y es excedida por una comunidad de vida que es reflejada en la "naturaleza" del ser humano, la "historia interna" de su existencia vivida e incorporada. Lo que hace una genuina comunidad no es el lenguaje sino la vida compartida. Como ha sido discutido con relación al descubrimiento de Freud del cuerpo humano, las historias del ser humano están enmarañadas con historias de animales y plantas al punto de la indistinción. El cuerpo humano no es un principio de clausura e inmunidad que requiere un suplemento defensivo de tecnicidad sino que más bien es el lugar de la vida compartida y de conexiones con todas las formas de vida, humanas y no humanas. Como lo ejemplifica la comprensión de Nietzsche de la vida de las plantas, incluida la planta humana, el cuerpo no tiene una identidad cerrada sobre sí misma que separa entre un interior y un exterior, entre formas de vida humanas y no humanas. Más bien, tal como lo concibe Nietzsche, el cuerpo humano está inserto en un medioambiente tal como una planta está enraizada en su suelo y así conectada a otras formas de vida de las cuales depende.

El homo natura de Nietzsche es concebido como una forma de vida que se ve alterada por su relación con otras formas de vida de las cuales depende profundamente. Como tal, el homo natura afirma la totalidad de la vida y la radical interrelación entre formas de vida humana y no humanas. Esta idea de una radical interrelación está en la base de la biopolítica afirmativa. En una biopolítica afirmativa, la política ya no designa un campo de asuntos humanos. Más bien, como sostiene Esposito apoyándose en la concepción de la voluntad de poder de Nietzsche, es la vida misma la que es siempre ya política.

que causa la descentración del sujeto humano son las estructuras lingüísticas no subjetivas de la significación. En mi hipótesis, la reciente emergencia del tema animal en la filosofía y las humanidades es sintomática de un descentramiento del sujeto humano que es nuevo en comparación con aquel que se puede encontrar en el primer y en el segundo debate sobre la crisis del humanismo. Más aún, este nuevo desarrollo depende de una recepción más reciente de la filosofía de Nietzsche, antes que de la continuación de la recepción del pensamiento de Heidegger. La filosofía de Nietzsche ofrece una perspectiva diferente a la de Heidegger sobre la crisis del humanismo ya que Nietzsche entiende la cultura humana sobre la base de la continuidad entre las formas de vida humana y animal. La crítica de Heidegger al humanismo, por el contrario, mantiene de principio a fin la discontinuidad entre las formas de vida humana y animal. Su crítica del humanismo tradicional busca en efecto proponer un nuevo humanismo (Sloterdijk 2006). En cambio, la aproximación de Nietzsche a la cultura, que considera la constitución de significado como algo que depende de la interacción con la animalidad del ser humano antes que con su trascendencia, abre nuevas maneras de pensar la comunidad de vida entre formas de vida humana y no humana.

Araucaria. Revista Iberoamericana de Filosofia, Politica, Humanidades y Relaciones Internacionales, año $23, \mathrm{n}^{\circ} 46$. Primer cuatrimestre de 2021. Pp. 291-306. ISSN 1575-6823 e-ISSN 2340-2199 https://dx.doi.org/10.12795/araucaria.2021.i46.14 
Finalmente, mi lectura del homo natura sugiere que existe una conexión entre el pensamiento de Nietzsche sobre la sexualidad y la comunidad de vida. Esto explicaría por qué la discusión de Nietzsche del homo natura termina con un discurso sobre sexualidad y política (MBM, 231-239). Esta conexión se apoya en el simultáneo descubrimiento de la reproducción sexuada de la vida en medio de las concepciones de la vida biológica del siglo XIX y de las relaciones sexuales y familiares como base de la reproducción social. Dado que según Nietzsche la vida es una fuerza pluralizadora y diversificadora, sostengo que es en la forma de una política sexual que la comunidad de vida expresa la política de la vida inherente a ella. De acuerdo a Nietzsche, es a través de las diferencias sexuales que la política de la vida altera y transforma las relaciones jurídico-políticas establecidas que se basan en convenciones artificiales y en contratos sociales. Es esta relación anti-fundacional entre vida y poder político lo que lleva a Esposito, siguiendo a Nietzsche, a refutar las categorías jurídico-políticas de la sociedad moderna tales como la libertad negativa o la igualdad formal, así como su discurso auto-legitimador sobre el contrato social según el cual el poder político es el resultado de individuos libres que dan su consentimiento a un pacto fundacional.

Es bien sabido que las así llamadas teorías agonistas de la democracia han cuestionado la búsqueda liberal del orden social basado en un acuerdo que permitiría traer paz y estabilidad a sus miembros individuales apelando a la concepción del poder en Nietzsche, para el cual no hay una superación final de la lucha, la competencia y la guerra, no hay una estabilidad final en un equilibrio de fuerzas definitivo, sino un conflicto y una lucha continua, productiva y abierta. Sin embargo, la teoría política del agonismo en general no ha articulado la teoría de la voluntad de poder de Nietzsche junto con su biopolítica afirmativa, basada en una comunidad de vida expandida más allá de lo humano. Según mi interpretación de la sección séptima de Más allá del bien y del mal, "Nuestras virtudes", es el propio Nietzsche el que señala el problema de la sexualidad como el locus decisivo en el que voluntad de poder y comunidad de vida se encuentran. Esta articulación se refleja en la creencia de Nietzsche en el antagonismo entre géneros debido a la inexistencia de la naturaleza sexual. Contra la fijación del devenir humano en una diferencia sexual naturalizada, Nietzsche sugiere considerar la sexualidad humana como la base para la transformación humana. Nietzsche emplea la sexualidad con objeto de des-esencializar el ser humano. Siguiendo la lectura de Derrida, la sexualidad en Nietzsche denota la alteridad de la naturaleza humana; una otredad en la naturaleza humana que ofrece a cada individuo los recursos para apartarse de y cuestionar los discursos civilizatorios sobre la verdad moral y metafísica que reflejan formas de poder sobre la naturaleza. Pero el discurso de Nietzsche sobre la sexualidad y su inevitable conflictividad también apunta 
hacia el rechazo de Foucault del sexo como "verdad oculta" del ser humano y del consiguiente empleo de la sexualidad como aparato de poder sobre la vida tal como se encuentra en la moderna scientia sexualis. A estas dos posiciones interpretativas, se tiene que añadir la creencia de Nietzsche de que el conflicto y la lucha sólo pueden ser productivos y fructíferos cuando las relaciones de poder reflejan un equilibrio: no puede haber lucha entre el débil y el fuerte, sino siempre únicamente entre fuerzas que son más o menos iguales. Aquí el equilibrio y la igualdad son el punto de partida para un conflicto productivo que permita la constitución de un orden jurídico que no se establezca como una forma política final y absoluta. Desde este punto de vista, es la interpretación feminista igualitaria del discurso de Nietzsche sobre la sexualidad la que tiene la última palabra, y lo que Nietzsche llama el "problema básico del hombre y la mujer" se vuelve el lugar para la construcción de sexualidades plurales como una práctica no-dominante y transformadora de ser humano. 


\section{Referencias bibliográficas:}

Agamben, Giorgio. Lo abierto. El hombre y el animal. Buenos Aires: Adriana Hidalgo, 2006.

Braidotti, Rosi. "Posthuman, All Too Human: Towards a New Process Ontology", Theory, Culture and Society, 23(7-8) (2006): 197-208.

Braidotti, Rosi. "Posthumanist Critical Theory", en Debashish Banerji y Makarand R. Paranjape (eds.), Critical Posthumanism and Planetary Futures, pp. 13-36. Nueva Dehli: Springer, 2016.

Braidotti, Rosi. Lo posthumano. Barcelona: Gedisa, 2015.

Deleuze, Gilles y Félix Guattari. Mil mesetas. Capitalismo y esquizofrenia.

Valencia: Pre-Textos, 2002.

Esposito, Roberto. Bios. Biopolítica y filosofía. Buenos Aires: Amorrortu, 2006. Foucault, Michel. Las palabras y las cosas. Méjico: Siglo XXI, 2007.

Haraway, Donna J. When Species Meet. Minneapolis y Londres: University of Minnesota Press, 2008.

Hayles, Katherine. How We Became Posthuman: Virtual Bodies in Cybernetics,

Literature and Informatics. Chicago y Londres: University of Chicago Press, 1999.

Heidegger, Martin. Carta sobre el humanismo. Madrid: Alianza, 2006.

Kant, Immanuel. Lógica. Madrid: Akal, 2000.

Kirby, Vicky. Quantum Anthropologies: Life at Large. Durham y Londres: Duke University Press, 2011.

Lemm, Vanessa. La filosofía animal de Nietzsche. Cultura, politica y animalidad del ser humano. Santiago de Chile: Ediciones UDP, 2010.

Lemm, Vanessa. "Is Nietzsche a Naturalist? Or How to Become a Responsible Plant", Journal of Nietzsche Studies, 47(1) (2016): 61-80.

Lemm, Vanessa. "Deconstructing the human: Ludwig Binswanger on Homo Natura in Nietzsche and Freud", en Daniel Conway (ed.), Nietzsche and The Antichrist: Religion, Politics, and Culture in Late Modernity. Londres y Nueva York: Bloomsbury, 2019.

MacKenzie, Adrian. Transductions: Bodies and Machines at Speed. Londres: Continuum, 2006.

Nietzsche, Friedrich. El anticristo: maldición sobre el cristianismo. Madrid: Alianza, 1997.

Nietzsche, Friedrich. La ciencia jovial. "La gaya scienza”. Caracas: Monte Ávila, 1999.

Nietzsche, Friedrich. Sobre la utilidad y los perjuicios de la historia para la vida. Madrid: Biblioteca Nueva, 1999.

Nietzsche, Friedrich. Más allá del bien y del mal. Madrid: Alianza, 2007. 
Nietzsche, Friedrich. Sobre verdad y mentira en sentido extramoral y otros fragmentos de filosofía del conocimiento. Madrid: Tecnos, 2012.

Plessner, Helmuth. Die Stufen des Organischen und der Mensch. Einleitung in die philosophische Anthropologie. Frankfurt: Suhrkamp Verlag, 2003.

Sloterdijk, Peter. Normas para el parque humano. Una respuesta a la Carta sobre el humanismo de Heidegger. Madrid: Siruela, 2006.

Tuncel, Yunus, ed. Nietzsche and Transhumanism: Precursor or Enemy. Cambridge: Cambridge Scholars, 2017.

van Ingen, Michiel. "Beyond the Nature/Culture Divide? The Contradictions of Rosi Braidotti's The Posthuman", Journal of Critical Realism, 15(5) (2016): 530-542.

Wolfe, Cary. What is Posthumanism? Minneapolis: University of Minnesota Press, 2010.

Wolfe, Cary. Before the Law: Humans and Other Animals in a Biopolitical Frame. Chicago y Londres: University of Chicago Press, 2013. 